\title{
Equilibrium existence in the circle model with linear quadratic transport cost ${ }^{\text {th }}$
}

\author{
M.A. de Frutos ${ }^{\mathrm{a}, *}$, H. Hamoudi ${ }^{\mathrm{b}}$, X. Jarque ${ }^{\mathrm{c}}$ \\ ${ }^{a}$ Departamento de Economia, Universidad Carlos III de Madrid, C/Madrid 126, 28903 Madrid, \\ Spain \\ ${ }^{\mathrm{b}}$ Universidad Europea de Madrid, Madrid, Spain \\ ${ }^{\mathrm{c}}$ Universitat Autonoma de Barcelona, Barcelona, Spain
}

Accepted 7 April 1999

\begin{abstract}
We treat the problem of existence of a location-then-price equilibrium in the circle model with a linear quadratic type of transportation cost function which can be either convex or concave. We show the existence of a unique perfect equilibrium for the concave case when the linear and quadratic terms are equal and of a unique perfect equilibrium for the convex case when the linear term is equal to zero. Aside from these two cases, there are feasible locations by the firms for which no equilibrium in the price subgame exists. Finally, we provide a full taxonomy of the price equilibrium regions in terms of weights of the linear and quadratic terms in the cost function.
\end{abstract}

Keywords: Hotelling; Circle; Equilibria

JEL classification: C72; D43

\section{Introduction}

Hotelling's (1929) model of spatial competition provides an appealing framework to address the nature of equilibrium in characteristic space and in geographic

\footnotetext{
The authors have benefited by comments and suggestions from X Martínez, Inés Macho and David Pérez-Castrillo

*Corresponding author Tel : +34-1-624-9600; fax: +34-1-624-9875

E-mail address: frutos@eco uc3m es (M A de Frutos)
} 
space. However, a major problem with the original model is that no configuration of prices and locations by the firm is an equilibrium. In particular, the Hotelling duopoly does not have equilibrium in prices for a wide range of locations by the firms. The source of this non-existence is the presence of a discontinuity in the seller's demand which appears at those prices where one seller can attract the whole market by slightly undercutting the price of his opponent (see d'Aspremont et al., 1979). But the non-existence of a pure price equilibrium is not due to this discontinuity itself, rather it is the failure of quasi-concavity of the profit function which causes the problem.

To circumvent the problem of non-existence of equilibrium, Hotelling's model has been thoroughly worked through in terms of altering the basic assumptions in the model. For example, various authors have considered different number of firms, alternative transport cost and demand formulations, non-uniform consumer densities over space, and many variations of the equilibrium concept. Contributions in this tradition have generally proposed specific convex transportation cost functions.

This line of research has been followed by d'Aspremont et al. (1979) who show the existence of an equilibrium for any location of the two firms when transportation costs are quadratic, i.e. $C(x)=a x^{2}, a>0$ where $x$ is the distance between firm and consumer. Then, Economides (1986) using the family of transportation cost functions $C(x)=x^{\alpha}, 1<\alpha<2$, showed that the region of existence of equilibrium is enlarged with the convexity of the consumers' utility function (price plus travel cost). Afterwards, Gabszewicz and Thisse (1986) showed that the combination of linear and quadratic terms in a convex transportation cost function, i.e., $C(x)=$ $a x+b x^{2}, a>0, b>0$, does not help to restore a price equilibrium. For this family of transportation costs Anderson (1988) has shown that when the solution concept is extended to allow for mixed strategies there is no symmetric equilibrium involving pure strategies if transport cost are not sufficiently convex. Moreover, he shows that the convexity of transport costs is crucial in determining whether there is excessive or insufficient diversity of products. More recently, Kats (1995) assumed the circumference of circle as the product space (a representation first used by Lerner and Singer, 1937) and has showed existence of equilibrium in the modified model where transportation costs are assumed to be linear in the distance. The author points out that the non-existence of equilibrium in the original Hotelling's model does not result from the specification of linear transport costs but it can be attributed to the fact that neither firm faces rivals on both sides of its location.

Taking into account the above results, we here analyze the price and location

\footnotetext{
${ }^{1}$ For some locations of the firms there is no equilibrium in pure strategies of the price subgame in this modified model At those locations, he considers the price equilibrium in mixed strategies But, if strategies are restricted to be pure, then there is no equilibrium in the price subgame if the distance between the firms is smaller than $1 / 4$
} 
decisions of duopolists in a circle model when transportation costs are assumed to be linear-quadratic, i.e. $C(x)=a x+b x^{2}$ where $a>0, b \in R$. This specification allows us to study how, if at all, the firm's competitive decisions are modified by the introduction of concave transportation cost as compared with convex ones. We show that both cases are strongly related via a change of parameters. In particular, taking concave transportation costs with $a=-b$, we show the existence of a symmetric equilibrium in pure strategies. Thus, in the circle model for a symmetric equilibrium to exist, it is not required transport cost to be sufficiently convex. Indeed, only two cost functions in the linear-quadratic family ensure existence of a perfect equilibrium in pure strategies. These are $C(x)=b x^{2}$ and $C(x)=b x-b x^{2}$ where $b>0$. For any other cost function in that family there are feasible locations by the firms for which no equilibrium in pure strategies in the price subgame exists. However, contrary to the linear market case, the non-existence of a pure price equilibrium occurs for locations that are too close together. Furthermore, at any given pair of locations, each merchant gains (or does not lose) from moving away as far as possible from the other. ${ }^{2}$ Finally, using only the convex linear quadratic transport costs, we compute exactly the regions of price equilibrium and study how they expand with the relative weight of the quadratic and linear terms in the cost functions. The regions of price equilibrium in the concave case (with concave transportation costs) can be obtained, easily, from the previous computations using a change of parameters.

The paper is organized as follows. Section 2 contains the model. Section 3 is devoted to the study of existence of a perfect equilibrium. In Section 4 we provide a full taxonomy of the regions of existence of equilibrium in the price subgame. Section 5 concludes. Finally, some of the proofs are contained in Appendix A.

\section{The model}

In a circular market of circumference of length 1 there are two sellers located at $x_{1}$ and $x_{2}, x_{1}, x_{2} \in[0,1)$, who charge mill prices $p_{1}$ and $p_{2}$, respectively. Both sellers supply a homogeneous product at zero marginal cost. A continuum of consumers are spread uniformly with unit density on the circumference. Each consumer purchase one unit of good. A consumer located at $x, x \in[0,1)$, purchasing a product from seller $i$ pays the cost of transporting the good from $x_{i}$ to $x$. Consumers will buy from the seller with the lower delivered or full price, mill price plus transportation cost. Transportation costs are assumed to be non-negative, increasing, and linear quadratic in the (relative) distance. Here we consider two possible transportation cost functions: $C^{+}(z ; a, b)=a z+b z^{2}, a \geq 0, b>0$, and

\footnotetext{
${ }^{2}$ For the linear market Anderson (1988) shows that equilibrium in prices may exist when firm locations are close but not when they are slightly further apart; see Proposition 2 in his paper
} 
$C^{-}(z ; a, b)=a z-b z^{2}, a \geq 0, b>0$. Notice that $C^{+}$is a convex function whereas $C^{-}$is concave.

The two-stage location-price game is modelled as follows: In the first stage of the game the two sellers choose simultaneously locations $x_{1}$ and $x_{2}$ in the circumference. Without loss of generality we may assume $x_{1}=0$ and $0 \leq x_{2} \leq 1 / 2$. In the second stage they simultaneously set prices $p_{1}$ and $p_{2}$, at which they offer to sell their product to consumers.

For $p_{2}$, and $x_{2}$ given, if the transportation cost function is $C^{+}$then the demand of seller 1 is given by

$$
D_{1}^{+}\left(x_{2}, p_{1}, p_{2}\right)= \begin{cases}D_{11}^{+}=1 & \text { if } p_{1} \in\left(0, I_{1}\right] \\ D_{12}^{+}=\frac{(a+b)\left(p_{2}-p_{1}\right)}{2 b x_{2}\left(a+b\left(1-x_{2}\right)\right)}+\frac{b-a}{2 b} & \text { if } p_{1} \in\left[I_{1}, I_{2}\right] \\ D_{13}^{+}=\frac{(2 a+b)\left(p_{2}-p_{1}\right)}{2\left(a+b x_{2}\right)\left(a+b\left(1-x_{2}\right)\right)}+\frac{1}{2} & \text { if } p_{1} \in\left[I_{2}, I_{3}\right] \\ D_{14}^{+}=\frac{(a+b)\left(p_{2}-p_{1}\right)}{2 b x_{2}\left(a+b\left(1-x_{2}\right)\right)}+\frac{b+a}{2 b} & \text { if } p_{1} \in\left[I_{3}, I_{4}\right] \\ D_{15}^{+}=0 & \text { if } p_{1} \in\left[I_{4}, \infty\right)\end{cases}
$$

where $I_{1}=p_{2}-x_{2}\left(a+b\left(1-x_{2}\right)\right), I_{2}=p_{2}-x_{2}\left(a+b x_{2}\right), I_{3}=p_{2}+x_{2}\left(a+b x_{2}\right)$ and $I_{4}=p_{2}+x_{2}\left(a+b\left(1-x_{2}\right)\right)$. As usual, $D_{2}^{+}\left(x_{2}, p_{1}, p_{2}\right)=1-D_{1}^{+}\left(x_{2}, p_{1}, p_{2}\right)$ are the demand functions for firm 2 .

Let us denote by $D_{1}^{-}\left(x_{2}, p_{1}, p_{2}\right)$ to the demand of seller 1 when the transport cost function is $C^{-}$. In De Frutos et al. (1998) it is shown that for any convex function $C$ with $C(0)=0$ there exists a concave one $T$ with $T(0)=0$ such that $D_{i}^{C}\left(x_{2}, p_{1}, p_{2}\right)=D_{i}^{T}\left(x_{2}, p_{1}, p_{2}\right)$ where $i=1,2$, i.e. the demand of each firm is the same under $C$ and $T$. The intuition behind this result is based on the fact that $C$ behaves for small values as $T$ does for large values. For the linear quadratic case, this result implies that if $a$ and $b$ are real and positive with $a>b$ then $D^{-}\left(x_{2}, p_{1}, p_{2} ; a, b\right)=D^{+}\left(x_{2}, p_{1}, p_{2} ; a-b, b\right)$. Notice that the demand for the concave case follow from the demand in the convex case through a change in the parameter values in the cost function.

\section{Equilibrium}

We now study the subgame perfect equilibrium of the two-stage non-cooperative game in which firms select a position at the first stage and subsequently set their price.

We denote by $B_{i}\left(p_{i}, p_{j}\right)=p_{i} D_{i}\left(p_{i}, p_{j}\right), i \neq j$, the profit function of seller $i$, $i=1,2$. A Nash Equilibrium in the price subgame in which the location choice is fixed, is a pair $\left(p_{1}^{*}, p_{2}^{*}\right)$ such that $p_{1}^{*}$ maximizes $B_{1}\left(p_{1}, p_{2}^{*}\right)$ on $\mathbb{R}^{+}$and $p_{2}^{*}$ 
maximizes $B_{2}\left(p_{1}^{*}, p_{2}\right)$ on $\mathbb{R}^{+}$. Then, a subgame perfect price-location equilibrium is defined as a pair $\left[\left(p_{1}^{*}, 0\right),\left(p_{2}^{*}, x_{2}^{*}\right)\right]$ such that

(i) $p_{1}^{*}=p_{1}^{*}\left(0, x_{2}^{*}\right)$ and $p_{2}^{*}=p_{2}^{*}\left(0, x_{2}^{*}\right)$,

(ii) $B_{i}\left(x_{2}^{*}, p_{1}^{*}\left(0, x_{2}^{*}\right), p_{2}^{*}\left(0, x_{2}^{*}\right)\right) \geq B_{i}\left(x_{2}, p_{1}^{*}\left(0, x_{2}\right), p_{2}^{*}\left(0, x_{2}\right)\right) \quad \forall x_{2} \in[0,1] i=1,2$.

Anderson (1986) have proved that for a general transportation cost function, $C(z)$, which is strictly convex, increasing and satisfies $C(0)=0$, a necessary condition for the existence of a subgame perfect price-location equilibrium is $C^{\prime}(0)=0$. Thus, for a convex linear quadratic transport cost, a necessary condition for existence of equilibrium is that the linear term be zero. We now show that this condition is sufficient as well for the linear quadratic transport cost. Moreover, we now also give a necessary and sufficient condition for existence of a perfect equilibrium for the concave case.

Proposition 1. (i) If the transportation cost function is convex and linear quadratic there exists a subgame perfect price-location equilibrium if and only if $a=0$. Moreover, if $a=0$ then the equilibrium is unique and it is given by $x_{2}=1 / 2$ and $p_{1}^{*}=p_{2}^{*}=b / 4$.

(ii) If the transportation cost is concave and linear quadratic there exists a subgame perfect price-location equilibrium if and only if $a=b$. Moreover, if $a=b$ then the equilibrium is unique and it is given by $x_{2}=1 / 2$ and $p_{1}^{*}=p_{2}^{*}=$ $b / 4$.

Proof. (i) Anderson (1986) has shown that $a=0$ is a necessary condition for a subgame perfect equilibrium to exist. When $a=0$ the demand functions are given by

$$
\begin{aligned}
& D_{1}^{+}\left(x_{2}, p_{1}, p_{2}\right) \\
& = \begin{cases}1 & \text { if } p_{1} \leq p_{2}-b\left(x_{2}-x_{2}^{2}\right) \\
\frac{\left(p_{2}-p_{1}\right)}{2 b x_{2}\left(1-x_{2}\right)}+\frac{1}{2} & \text { if } p_{1} \in\left[p_{2}-b\left(x_{2}-x_{2}^{2}\right), p_{2}+b\left(x_{2}-x_{2}^{2}\right)\right] \\
0 & \text { if } p_{1} \geq p_{2}+b\left(x_{2}-x_{2}^{2}\right)\end{cases}
\end{aligned}
$$

and $D_{2}^{+}\left(x_{2}, p_{1}, p_{2}\right)=1-D_{1}^{+}\left(x_{2}, p_{1}, p_{2}\right)$. Thus, the profit functions are strictly concave and for any given location by firm $2, x_{2} \in[0,1 / 2]$, there exists a unique pair of equilibrium prices, $p_{1}^{*}=p_{2}^{*}=b x_{2}\left(1-x_{2}\right)$. Now, easy computations show that there exists a unique subgame perfect price-location equilibrium at $x_{2}=1 / 2$ and $p_{1}^{*}=p_{2}^{*}=b / 4$. (ii) The second part of this result follows easily from the fact that $D^{-}\left(x_{2}, p_{1}, p_{2} ; a, b\right)=D^{+}\left(x_{2}, p_{1}, p_{2} ; a-b, b\right)$ and part (i). 
In the circle model the existence of equilibrium in pure strategies does not rely on how convex is the transportation cost function. Rather than this, in order to ensure the existence of equilibrium we need the cost function to be either sufficiently convex or sufficiently concave. What is special about the linear case? If the transportation cost is linear consumers care as much for the price they have to pay as for the distance they have to travel. Furthermore, firms perceive price and location as two strategic substitutes. Thus, for some locations by firm 2 , it is possible and profitable for firm 1 to undercut its price by exactly $x_{2}$ to capture the whole market. This undercutting price increases as $x_{2}$ decreases. In the other extreme cases $\left(C(z)=b z^{2}\right.$ and $\left.C(z)=b\left(z-z^{2}\right)\right)$ location and price are strategic complements, i.e. each firm reacts to increases in the distance between the firms by increasing its equilibrium price. Hence, when firms are very close to each other the equilibrium price is so small that no firm has incentives to undercut it so as to capture the entire market.

\section{Equilibrium regions}

The non-existence of a subgame perfect equilibrium in our model of product differentiation can be attributed to the non-concavity of the profit functions. More precisely, we will show that the profit function is double-peaked so that for some locations of the firms there is no price equilibrium.

Let seller 1 be located at 0 and let seller 2 be located at $x_{2}$. Furthermore, let us assume that $C(z)=C^{+3}$. Anderson (1986) showed that if a price equilibrium exists for a given location $x_{2}$ it has to be given by

$$
p_{1}^{*}=p_{2}^{*}=p^{*}\left(x_{2}\right)=\frac{\left(a+b x_{2}\right)\left(a+b\left(1-x_{2}\right)\right)}{2 a+b}
$$

Notice that if an equilibrium exists it has to be symmetric. Therefore, $B_{i}\left(x_{2}, p_{1}^{*}, p_{2}^{*}\right)=0.5 p^{*}\left(x_{2}\right)$, for $i=1,2$. Moreover, looking at $p^{*}\left(x_{2}\right)$ it is straightforward to see that $x_{2}=0.5$ is the unique maximum of $p^{*}\left(x_{2}\right)$ whenever $b \neq 0$.

Since we have explicit expressions of the profit functions we can study the regions of equilibrium (i.e. the range of $x_{2}$ for which an equilibrium in the price subgame exists). We can also study how these regions change with the parameter values of the transport cost function. More precisely, we will show that these regions go from $x_{2} \geq 1 / 4$ to $x_{2} \geq 0$ as we go from $C(z)=a z$ to $C(z)=b z^{2}$ through the family $C(z)=a z+b z^{2}$. The following proposition contains these results.

Proposition 2. Let $C(z)=C^{+}(z)=a z+b z^{2}$ and let $\alpha=b / a$. The regions of price equilibrium are (see Fig. 1)

\footnotetext{
${ }^{3}$ Notice that the results for $C(z)=C^{-}$can be derived from the results in the convex case by using the change in the parameter values introduced in Section 2
} 
(i) $\left[z_{4}(\alpha), \frac{1}{2}\right]$ if $0 \leq \alpha \leq 1$.

(ii) $\left[z_{3}(\alpha), \frac{1}{2}\right]$ if $\alpha>1$,

where $z_{4}(\alpha)=\frac{1}{(4+\alpha)}$ and $z_{3}(\alpha)$ is given by

$$
z_{3}(\alpha)= \begin{cases}\frac{-\left(1+2 \alpha+\alpha^{2}\right)+\left(2+3 \alpha+\alpha^{2}\right) \sqrt{\alpha}}{2\left(\alpha^{3}+3 \alpha^{2}+2 \alpha-1\right)} & \text { if } 2\left(\alpha^{3}+3 \alpha^{2}+2 \alpha-1\right) \neq 0 \\ \frac{1}{4} & \text { if } 2\left(\alpha^{3}+3 \alpha^{2}+2 \alpha-1\right)=0 .\end{cases}
$$

Proof. See Appendix A.

Remark 1. We can observe from the expressions for $z_{4}(\alpha)$ and $z_{3}(\alpha)$ that if $\alpha \rightarrow \infty$ $(b>0, a \rightarrow 0)$ then the price equilibrium region tends to the interval $\left[0, \frac{1}{2}\right]$. On the other hand if $\alpha \rightarrow 0(a>0, b \rightarrow 0)$ then the price equilibrium region tends to the interval $\left[\frac{1}{4}, \frac{1}{2}\right]$. These results correspond to the limit cases of the quadratic transportation cost $(a=0)$ and linear transportation cost $(b=0)$. Furthermore, we observe that the regions of price equilibrium change monotonically.

\section{Conclusions}

We have shown that for our family of transportation costs the results for the concave case follow from the result in the convex case through a change in the parameter values in the cost function. Consequently, there exists a perfect location-price equilibrium in the circle model if $C^{-}(z)=b z-b z^{2}$ that corresponds to the equilibrium for the cost function $C^{+}(z)=b z^{2}$. This equilibrium involves maximal differentiation. This result implies that the existence of a perfect equilibrium in the circle model is not related to the convexity of the cost function.

In the circle model, as compare with the linear model, firms face symmetric conditions: each faces a rival on both sides of its location. This symmetry is in the basis for the equivalence result between the concave transport cost and the convex one. Moreover, if transportation costs are not linear then each firm is strictly better off the further away is from its rival. Thus, in the circle model the unique pattern of product differentiation is always maximal differentiation. If transportation costs are linear this pattern is an equilibrium as well but there are other equilibria which involve less differentiation between the firms.

One solution to the non-existence problem is to impose location restrictions between the firms, in particular, to impose a minimal distance constraint between them. We have shown that this distance must go from $1 / 4$ (for the linear transport cost case) to 0 (for the quadratic transport case). Another solution is to allow for mixed strategies in the price subgame. However, mixed strategies solutions demand great computational ability of the agents and may seem implausible. 


\section{Acknowledgements}

Financial support from the DIGYCIT, under project PB96-0118, is gratefully acknowledged.

\section{Appendix A. Proof of Proposition 2}

Let seller 1 be located at 0 and let seller 2 be located at $x_{2}$. From Anderson (1986) it is known that if a price equilibrium exists for a given location $x_{2}$ it has to be given by

$$
p_{1}^{*}=p_{2}^{*}=\frac{\left(a+b x_{2}\right)\left(a+b\left(1-x_{2}\right)\right)}{2 a+b}
$$

Consequently, a price equilibrium exists when seller 2 is located at $x_{2}$ if and only if

$$
\begin{aligned}
& B_{1}\left(x_{2}, p_{1}^{*}, p_{2}^{*}\right) \geq B_{1}\left(x_{2}, p_{1}, p_{2}^{*}\right) \forall p_{1} \\
& B_{2}\left(x_{2}, p_{1}^{*}, p_{2}^{*}\right) \geq B_{2}\left(x_{2}, p_{1}^{*}, p_{2}\right) \forall p_{2}
\end{aligned}
$$

Let us confine our analysis to seller 1 . If seller 2 charges $p_{2}^{*}$ the profit function of seller 1 is

$$
B_{1}^{+}\left(x_{2}, p_{1}, p_{2}^{*}\right)= \begin{cases}B_{11}^{+}=p_{1} & \text { if } p_{1} \in\left(0, I_{1}^{*}\right] \\ B_{12}^{+}=\frac{p_{1}(a+b)\left(p_{2}^{*}-p_{1}\right)}{2 b x_{2}\left(a+b\left(1-x_{2}\right)\right)}+\frac{p_{1}(b-a)}{2 b} & \text { if } p_{1} \in\left[I_{1}^{*}, I_{2}^{*}\right] \\ B_{13}^{+}=\frac{p_{1}(2 a+b)\left(p_{2}^{*}-p_{1}\right)}{2\left(a+b x_{2}\right)\left(a+b\left(1-x_{2}\right)\right)}+\frac{p_{1}}{2} & \text { if } p_{1} \in\left[I_{2}^{*}, I_{3}^{*}\right] \\ B_{14}^{+}=\frac{p_{1}(a+b)\left(p_{2}^{*}-p_{1}\right)}{2 b x_{2}\left(a+b\left(1-x_{2}\right)\right)}+\frac{p_{1}(b+a)}{2 b} & \text { if } p_{1} \in\left[I_{3}^{*}, I_{4}^{*}\right] \\ B_{15}^{+}=0 & \text { if } p_{1} \in\left[I_{4}^{*}, \infty\right)\end{cases}
$$

where $I_{1}^{*}=p_{2}^{*}-x_{2}\left(a+b\left(1-x_{2}\right)\right), I_{2}^{*}=p_{2}^{*}-x_{2}\left(a+b x_{2}\right), I_{3}^{*}=p_{2}^{*}+x_{2}\left(a+b x_{2}\right)$ and $I_{4}^{*}=p_{2}^{*}+x_{2}\left(a+b\left(1-x_{2}\right)\right)$.

It is easy to see that $B_{1 i}^{+}\left(x_{2}, p_{1}, p_{2}^{*}\right), i=2,3,4$, are strictly concave functions in the variable $p_{1}$ and that $B_{14}^{+}\left(x_{2}, p_{1}, p_{2}^{*}\right)$ is a strictly decreasing function on $\left[I_{3}^{*}, I_{4}^{*}\right]$. Thus, the profit function can have at most two local maxima: one lying on $\left[I_{1}^{*}, I_{2}^{*}\right]$ and another on $\left[I_{2}^{*}, I_{3}^{*}\right]$. The first derivative of $B_{12}^{+}\left(x_{2}, p_{1}, p_{2}^{*}\right)$ has a zero at

$$
p_{1}=\bar{p}_{1}=\frac{\left(a+b\left(1-x_{2}\right)\right)\left[a(a+b)+2 x_{2}\left(-a^{2}+a b+b^{2}\right)\right]}{2(2 a+b)(a+b)}
$$


Notice that $\bar{p}_{1}$ may not always lie on $\left[I_{1}^{*}, I_{2}^{*}\right]$. Finally, the first derivative of $B_{13}^{+}\left(x_{2}, p_{1}, p_{2}^{*}\right)$ gives a local maximum at $p_{1}=p_{1}^{*}$ which always lies on $\left[I_{2}^{*}, I_{3}^{*}\right]$. Therefore, all the possible graphics of $B_{1}^{+}\left(x_{2}, p_{1}, p_{2}^{*}\right)$ for which $p_{1}=p_{1}^{*}$ is a global maximum are those drawn in Fig. 2.

We now discuss each case separately to obtain conditions on $x_{2}$ that will ensure the existence of an equilibrium in prices.

Case 1. See Fig. $2 ; \bar{p}_{1} \in\left[I_{1}^{*}, I_{2}^{*}\right]$ and $B_{13}^{+}\left(x_{2}, p_{1}^{*}, p_{2}^{*}\right) \geq B_{12}^{+}\left(x_{B}, \bar{p}_{1}, p_{2}^{*}\right)$.

For $\bar{p}_{1}$ to lie on this interval the following two conditions must hold:

$$
\begin{aligned}
& \bar{p}_{1}=p_{2}^{*}+x_{2}\left(\left(a+b\left(1-x_{2}\right)\right) \geq 0\right. \\
& \bar{p}_{1}-p_{2}^{*}+x_{2}\left(a+b x_{2}\right) \leq 0
\end{aligned}
$$

After some computations it is possible to show that conditions above are satisfied if and only if the following two conditions hold:

$$
\begin{aligned}
& 2\left(1+3 \alpha+\alpha^{2}\right) x_{2}-(1+\alpha) \geq 0 \\
& 2 \alpha\left(3+3 \alpha+\alpha^{2}\right) x_{2}^{2}+\left(1+5 \alpha+\alpha^{2}\right) x_{2}-(1+\alpha)^{2} \leq 0
\end{aligned}
$$

Since $B_{13}^{+}\left(x_{2}, p_{1}^{*}, p_{2}^{*}\right) \geq B_{12}^{+}\left(x_{B}, \bar{p}_{1}, p_{2}^{*}\right)$ must also hold we get the following additional condition

$$
4\left(-1+2 \alpha+3 \alpha^{2}+\alpha^{3}\right) x_{2}^{2}+4(1+\alpha)^{2} x_{2}-(1+\alpha)^{2} \geq 0
$$

Since $\alpha$ is positive it is possible to get the regions where conditions Eq. (A.1) and Eq. (A.2) are satisfied. On the other hand, by studying the zeroes of the polynomial in $x_{2}$ in condition Eq. (A.3) (taking into account the possible zero in the coefficient of the quadratic term) it is deduced that Eq. (A.3) is always satisfied

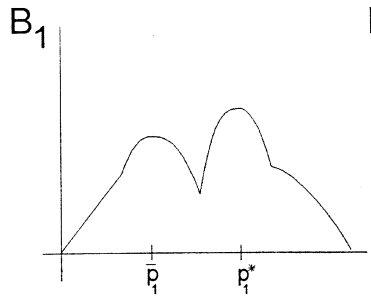

Case 1

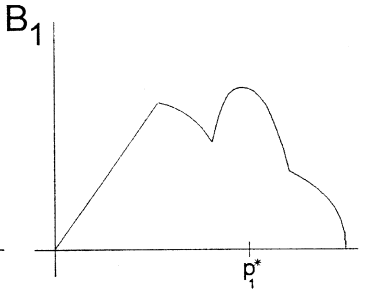

Case 2

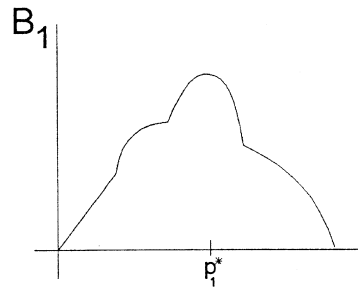

Case 3

Fig 2 Profit functions 
when $x_{2}$ is bigger than $z_{3}(\alpha)$, where $z_{3}(\alpha)$ is the zero of the polynomial when the coefficient of the quadratic term is not zero, i.e. $\left(-1+2 \alpha+3 \alpha^{2}+\alpha^{3}\right) \neq 0$. Finally, we show that $z_{3}(\alpha)$ is smaller than $z_{2}(\alpha)$. Based on this considerations it is straightforward to show that the conditions of Eqs. (1)-(3) hold simultaneously if and only if the two following conditions hold:

$$
\begin{aligned}
& x_{2} \in\left[z_{1}(\alpha), z_{2}(\alpha)\right] \text { if } 0 \leq \alpha \leq 1 \\
& x_{2} \in\left[z_{3}(\alpha), z_{2}(\alpha)\right] \text { if } \alpha>1
\end{aligned}
$$

where $z_{3}(\alpha)$ is given in Proposition 2 and

$$
\begin{aligned}
& z_{1}(\alpha)=\frac{1+\alpha}{2\left(1+3 \alpha+\alpha^{2}\right)} \\
& z_{2}(\alpha)=\frac{-\left(2+5 \alpha+3 \alpha^{2}\right)+\left(2+3 \alpha+\alpha^{2}\right) \sqrt{1+8 \alpha}}{4 \alpha\left(3+3 \alpha+\alpha^{2}\right)}
\end{aligned}
$$

Case 2. See Fig. 2; $\bar{p}_{1} \notin\left[I_{1}^{*}, I_{2}^{*}\right] \quad$ with $\bar{p}<p_{2}^{*}-x_{2}\left(a+b\left(1-x_{2}\right)\right)$ and $B_{13}^{+}\left(x_{2}, p_{1}^{*}, p_{2}^{*}\right) \geq B_{12}^{+}\left(x_{2}, p_{2}^{*}-x_{2}\left(a+b\left(1-x_{2}\right), p_{2}^{*}\right)\right.$.

These two conditions hold if and only if:

$$
\begin{aligned}
& 2\left(1+3 \alpha+\alpha^{2}\right) x_{2}-(1+\alpha)<0 \\
& (4+\alpha) x_{2}-1 \geq 0
\end{aligned}
$$

The two conditions are satisfied simultaneously if and only if $\alpha \leq 1$ and $x_{2} \in$ $\left[z_{4}(\alpha), z_{1}(\alpha)\right]$, where $z_{4}(\alpha)=\frac{1}{(4+\alpha)}$.

Case 3. See Fig. $2 ; \bar{p}_{1} \notin\left[I_{1}^{*}, I_{2}^{*}\right]$ with $\bar{p}>p_{2}^{*}-x_{2}\left(a+b x_{2}\right)$. It is easy to see that this holds if and only if Eq. (A.2) does not hold. Therefore, there will be an equilibrium if $x_{2} \in\left[z_{2}(\alpha), \frac{1}{2}\right]$.

Thus, the price equilibrium regions are given by:

- If $0 \leq \alpha \leq 1$ they are

$$
\left[z_{1}(\alpha), z_{2}(\alpha)\right] \cup\left[z_{2}(\alpha), \frac{1}{2}\right] \cup\left[z_{4}(\alpha), z_{1}(\alpha)\right]=\left[z_{4}(\alpha), \frac{1}{2}\right],
$$

- If $\alpha>1$ they are

$$
\left[z_{3}(\alpha), z_{2}(\alpha)\right] \cup\left[z_{2}(\alpha), \frac{1}{2}\right]=\left[z_{3}(\alpha), \frac{1}{2}\right] .
$$




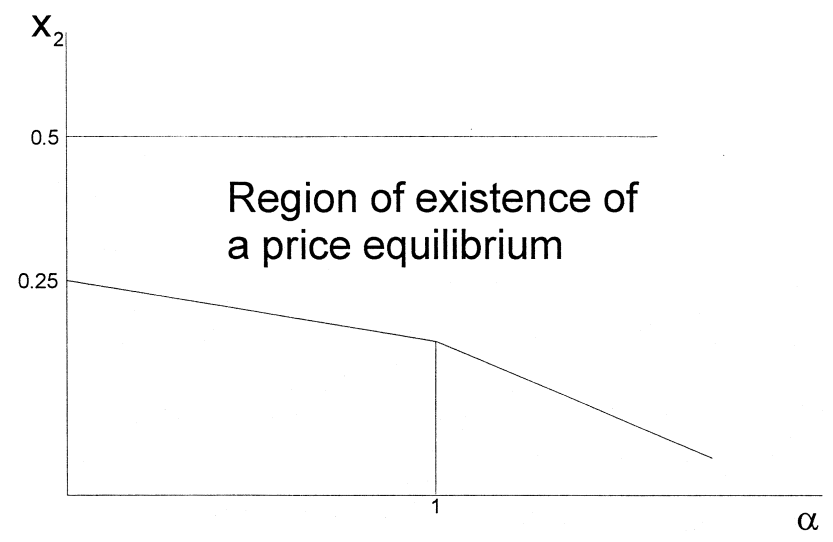

Fig. 1. Equilibrium regions.

Adding all these conditions together we get the result.

\section{References}

Anderson, S.P., 1986. Equilibrium existence in the circle model of product differentiation. London Papers in Regional Science Series 16, 19-29.

Anderson, S.P., 1988. Equilibrium existence in the linear model of spatial competition. Economica 55, 479-491.

D’Aspremont, C., Gabszewicz, J.J., Thisse, J.F., 1979. On Hotelling's stability in competition. Econometrica 47, 1145-1150.

De Frutos, M.A., Hamoudi, H., Jarque, X., 1988. Spatial competition and consumer theory, mimeo Universidad Carlos III.

Economides, N., 1986. Minimal and maximal product differentiation in Hotelling's duopoly. Economic Letters. 21, 67-71.

Gabszewicz, J.J., Thisse, J.F., 1986. On the nature of competition with differentiated products. Economic Journal 96, 160-172.

Hotelling, H., 1929. Stability in competition. Economic Journal 39, 41-57.

Kats, A., 1995. More on Hotelling's stability in competition. International Journal of Industrial Organization 13, 89-93.

Lerner, A.P., Singer, H.W., 1937. Some notes on duopoly and spatial competition. Journal of Political Economy. 45, 145-186. 